\title{
Ultraconserved regions in multiple sclerosis
}

\author{
European Journal of Human Genetics (2005) 13, 998-999. doi:10.1038/sj.ejhg.5201457; \\ published online 29 June 2005
}

In their recent publication, Bejerano et al ${ }^{1}$ identified 481 'ultraconserved elements' in the human genome. They defined these elements as genomic segments longer than $200 \mathrm{bp}$, showing 100\% sequence homology with rat and mouse, but excluding ribosomal RNA regions. Many of these elements also show extremely high levels of homology with the chicken and dog genomes. Overall, these elements are more highly conserved than protein coding regions. The mechanisms responsible for maintaining these sequences through evolution are unclear but seem likely to include profound negative selection, suggesting that these segments have important, if not vital, functions. Whether these functions will necessarily be relevant to the pathogenesis of multiple sclerosis (MS) is speculative.

The high levels of homology are reflected in correspondingly low levels of variation within the general human population. Bejerano et $a l^{1}$ identified just six validated single-nucleotide polymorphisms (SNPs) lying within these ultraconserved elements (rs1538101, rs1861100, rs2056116, rs7092999, rs7143938 and rs9572903), a total of $106767 \mathrm{bp}$ of sequence.

While there remains limited data on the functional effects of SNPs within ultraconserved regions, several pathogenic mutations in the region that regulates sonic hedgehog $(\mathrm{SHH})$ expression are associated with preaxial polydactyly, a congential limb malformation. ${ }^{2}$ Given the likely biological importance of these ultraconserved regions, it is reasonable to infer that these six variants have a high prior probability of conferring functional significance; however, the nature of this altered function is currently unpredictable. We tested these six SNPs from ultraconserved elements for evidence of association with
MS, in which genetic factors are implicated, ${ }^{3}$ but their nature and identity are largely unknown. Association was sought by typing each variant in 938 trio families (an affected individual and both parents). All patients satisfied Poser diagnostic criteria ${ }^{4}$ with the majority of patients having relapsing-remitting disease $(64.8 \%), 28.7 \%$ were secondary progressive and $6.5 \%$ were primary progressive.

These assays were performed using TaqMan methodology $^{5}$ (the primers used are listed in Supplementary Table S1) on a 7900HT Sequence Detection System, according to the manufacturer's standard conditions. Marker rs9572903 was found to be monomorphic. Results for the other five SNPs, analysed using the TRANSMIT program ${ }^{6}$ to search for evidence of transmission distortion, are shown in Table 1.

In each case, 163 samples were typed in duplicate to ensure genotyping accuracy; no inconsistency was observed for any marker. Only one Mendelian inconsistency was observed across all five markers, indicating an approximate genotyping error rate of $0.02 \%$. None of the markers showed deviation from Hardy-Weinberg equilibrium. After appropriate Bonferroni correction, none of the SNPs show any statistically significant evidence for association. SNP rs7143938 showed reduced transmission of the minor allele, which just reached nominal significance and therefore could reflect a modest effect. This SNP lies in the second intron of the Mirror Image Polydactyly Gene 1 (MIPOL1) on chromosome 14q13. This is a developmental gene, and it is not inconceivable that the gene product influences the immune or nervous systems. The observed under-transmission could indicate negative selection of the minor allele but, if so, it is unlikely that this allele would have reached a population frequency of

Table 1 SNPs from ultraconserved regions in multiple sclerosis

\begin{tabular}{|c|c|c|c|c|c|c|}
\hline Marker & Gene name & Chromosome location & Genotyping rate $e^{\mathrm{a}}(\%)$ & Heterozygosity (\%) & $\begin{array}{c}\text { Minor allele } \\
\text { frequency (\%) }\end{array}$ & $\begin{array}{c}\text { Uncorrected }^{\mathrm{b}} \\
\text { P-value }^{\mathrm{b}}\end{array}$ \\
\hline rs1538101 & $\mathrm{BNC2}$ & $9 p 22.2$ & 92.7 & 14.0 & 7.2 & 0.98 \\
\hline rs2056116 & Intergenic & $4 p 15.33$ & 96.7 & 48.3 & 39.4 & 0.79 \\
\hline rs7092999 & Intergenic & $10 q 24.31$ & 98.7 & 49.5 & 41.3 & 0.69 \\
\hline rs7143938 & MIPOL1 & $14 \mathrm{q} 13.3$ & 92.6 & 43.1 & 31.8 & 0.035 \\
\hline
\end{tabular}

${ }^{\mathrm{a}}$ The proportion of potential genotypes successfully recorded.

${ }^{b}$ After Bonferroni correction none of these results are statistically significant. 
$>30 \%$ in the face of such negative selection. It is more likely that the evidence for under-transmission is due to sampling as suggested by the lack of significance after Bonferroni correction.

In summary, we found no evidence that variation in these ultraconserved regions influences susceptibility to MS.

\section{Acknowledgements}

We thank members of the Association of British Neurologists for notifying families. This work was supported by the Wellcome Trust (Grant 057097), the Multiple Sclerosis Society of the United States (Grant RG3500-A-1), and the Multiple Sclerosis Society of Great Britain and Ireland (Grant 730/02).

\footnotetext{
Maria Ban ${ }^{1}$, Mel Maranian ${ }^{1}$, Tai Wai Yeo ${ }^{1}$, Julia Gray ${ }^{1}$, Alastair Compston ${ }^{1}$ and Stephen Sawcer ${ }^{1}$

${ }^{1}$ University of Cambridge Neurology Unit, Department of Clinical Neurosciences, Addenbrooke's Hospital, Level 6, 'A'
}

Block, Box 165, Hills Road, Cambridge CB2 2QQ, UK. E-mail:mb531@medschl.cam.ac.uk

\section{References}

1 Bejerano G, Pheasant M, Makunin I et al: Ultraconserved elements in the human genome. Science 2004; 304: 1321-1325.

2 Lettice LA, Heaney SJ, Purdie LA et al: A long-range Shh enhancer regulates expression in the developing limb and fin and is associated with preaxial polydactyly. Hum Mol Genet 2003; 12: $1725-1735$.

3 Hogancamp WE, Rodriguez M, Weinshenker BG: The epidemiology of multiple sclerosis. Mayo Clin Proc 1997; 72: 871-878.

4 Poser CM, Paty DW, Scheinberg L et al: New diagnostic criteria for multiple sclerosis: guidelines for research protocols. Ann Neurol 1983; 13: 227-231.

5 Holland PM, Abramson RD, Watson R, Gelfand DH: Detection of specific polymerase chain reaction product by utilizing the $5^{\prime}-3^{\prime}$ exonuclease activity of Thermus aquaticus DNA polymerase. Proc Natl Acad Sci USA 1991; 88: 7276-7280.

6 Clayton D: A generalization of the transmission/disequilibrium test for uncertain-haplotype transmission. Am J Hum Genet 1999; 65: $1170-1177$.

Supplementary Information accompanies the paper on European Journal of Human Genetics website (http://www.nature.com/ejhg) 